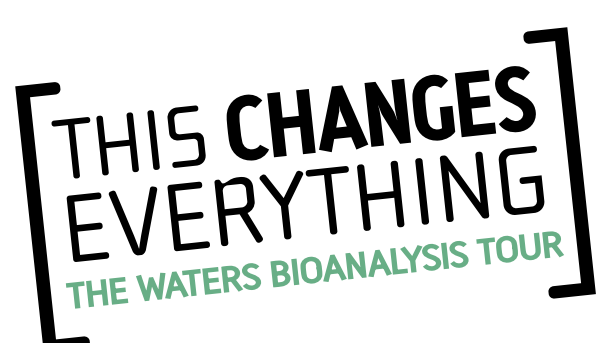

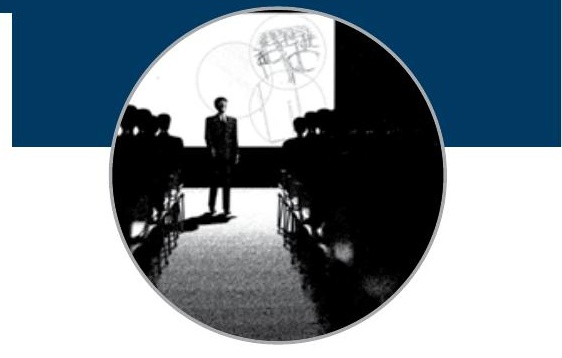

\section{The Waters Bioanalysis World Tour: Insights into the Next Decade of Regulated Bioanalysis}

\begin{abstract}
International Pharmaceutical Federation Pharmaceutical Science World Congress and American Association of Pharmaceutical Scientists Annual Meeting and Exposition, New Orleans, LA, USA, I5 November 2010.

Continuing the Waters Corporation Bioanalysis World Tour, an evening symposium was held aboard the Creole Queen (New Orleans, LA, USA) on 15 November 2010 as part the combined International Pharmaceutical Federation Pharmaceutical Science World Congress and the American Association of Pharmaceutical Scientists Annual Meeting and Exposition. The symposium was chaired by Warren Potts (Waters Corporation, MA, USA) and Stephen Lowes (Advion BioServices Inc., NY, USA). The symposium looked to the future, with three expert speakers focusing on the next 10 years of regulated bioanalysis. Walter Korfmacher (Merck, NJ, USA) discussed the use of high-resolution MS in discovery absorption, distribution, metabolism and excretion (ADME); Gary Schultz (Advion BioServices Inc.) covered integrated solutions for the future of bioanalysis and Paul Rainville (Waters Corporation) spoke about microfluidic exact-mass LC-MS/MS analysis for small-molecule biomarker and pharmaceutical quantitation. Following the presentations, all the speakers took questions from the audience and continued lively discussion over a dinner reception.
\end{abstract}

Keywords: bioanalysis $\approx$ discovery $₫$ exact mass $₫$ future $\approx$ high-resolution $M S \backsim$ integrated $\approx$ microfluidic $\approx$ regulated

\section{Speaker profiles}

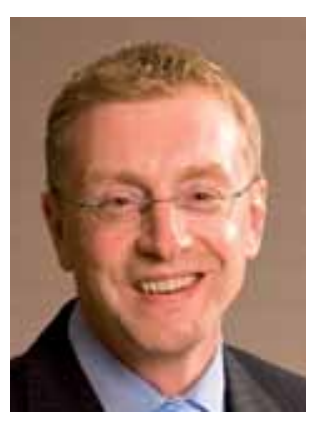

- Stephen Lowes, Advion BioServices Inc., NY, USA. Chair Stephen Lowes joined Advion in 1995 and has directed the scientific operations of Advion BioServices Inc. since 1997. Prior to joining Advion, he worked for VG Biotech (Manchester, UK), where he had roles in quadrupole mass spectrometer product development and became the demonstration laboratory manager. Since joining Advion, Steve has focused on quantitative LC-MS/MS analysis of small molecules using atmospheric pressure ionization techniques on quadrupole instruments. His current initiatives include expanding the bioanalytical services of Advion in the regulated and nonregulated areas of drug discovery and development, and addressing biotherapeutics and large-/small-molecule biomarkers with ligand-binding and novel LC-MS approaches. Steve is actively involved with the American Association of Pharmaceutical Scientists (AAPS) and various industry committees and focus groups that address current topics in regulated bioanalysis. He is a past chair of the AAPS bioanalytical focus group and a regular speaker at national and international conferences.

\section{Ryan De Vooght-Johnson}

Author for correspondence:

Bioanalysis, Commissioning Editor, Future Science Group, London, N3 IQB, UK

E-mail: r.devooght-johnson@

future-science.com

\section{FUTURE
SCIENCE}




\section{Introduction}

The Waters bioanalysis world tour $[1,101]$ continued with an evening symposium, held on board the Creole Queen, New Orleans (LA, USA) as part of the combined International Pharmaceutical Federation Pharmaceutical Science World Congress and the American Association of Pharmaceutical Scientists Annual Meeting and Exposition [102]. Warren Potts (Waters Corporation) co-chaired the event with Stephen Lowes (Advion).

Lowes started the proceedings with a brief introduction and highlighted why now is the most exciting time to be a bioanalyst since the advent of atmospheric pressure ionization techniques with triple quadrupole instruments 25 years ago. Clearly the bar is set very high, but the topics discussed in this symposium confirmed this with scientific rationale. Several factors make the current bioanalysis environment particularly special - the deconstruction of the traditionally western-centric pharmaceutical industry and its reconstruction in a global manner, patent expirations, the rise of biotherapeutics (or the fall of small molecules) and the need to do more (in terms of sample numbers, analytical confidence and drug safety) with less money. These factors may sound frightening to some, but they drive exciting opportunities for scientists to step forward and lead developments of technology and the application to regulated bioanalysis.

"...now is the most exciting time to be a bioanalyst since the advent of atmospheric pressure ionization techniques with triple quadrupole instruments 25 years ago."

With the globalized pharmaceutical market, there is an ongoing evolution in regulatory expectations and requirements. The US FDA are now joined by the European Medical Agency in drafting bioanalytical method validation guidance. Simultaneously, bioanalytical scientists are presenting the attractive potential of new strategies and technologies such as dried blood spot (DBS) sample collection and high-resolution MS (HRMS). The next few years of regulated bioanalysis are very likely to see the advent of great improvements and innovative science driven by the needs of the pharmaceutical industry.

\section{The potential for HRMS to be used as a tool for discovery absorption, distribution, metabolism \& excretion quantitative assays}

Walter Korfmacher, Exploratory Drug Metabolism, Merck Research Laboratories, NJ, USA

Walter Kormacher's talk focused on the impending paradigm shift from using LC-MS/MS to using HRMS for meeting the ever-increasing demand for higher-throughput assays for compounds in the drug-discovery stage for the analysis of discovery samples obtained from various absorption, distribution, metabolism, excretion (ADME) and pharmacokinetic (PK) studies.

Korfmacher started by illustrating the new pipeline for drug discovery (FIgure I). The chemists on a large pharmaceutical site may develop 100-200 new compounds a week, all of which need separate in vitro ADME screens and in vivo PK screens to be carried out by discovery bioanalysis. The current tool of choice for these assays is triple quadrupole LC-MS/MS. However, the new generation of HRMS instruments have advantages that make them likely to replace LC-MS/MS: no method development needed for molecular mass of 100-1000 and a linear or dynamic range of three to four orders of magnitude and information on metabolites can be captured in the same run at no extra cost. The metabolite information will be

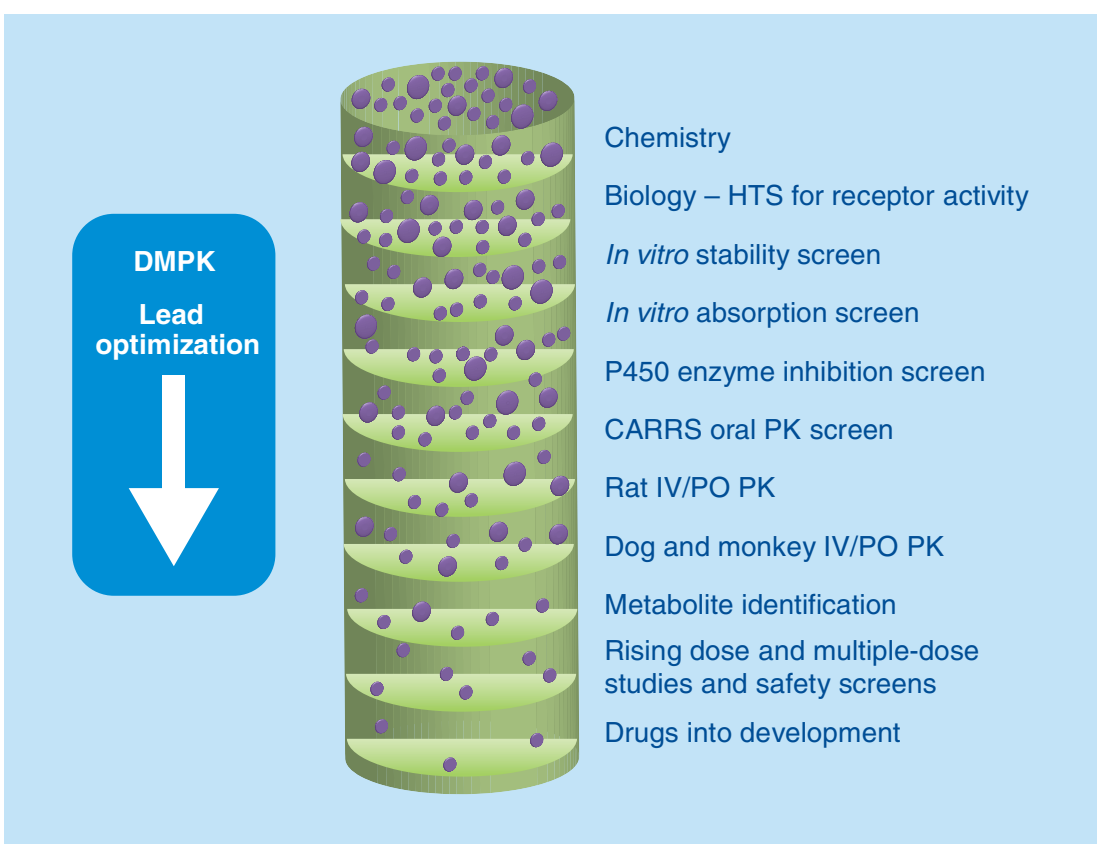

Figure 1. New drug discovery pipeline.

DMPK: Drug metabolism/pharmacokinetics; HTS: High-throughput screening; IV: Intravenous; PO: Per os. 


\section{News \& ANalysis | Waters BIoAnalysis World TOUR}

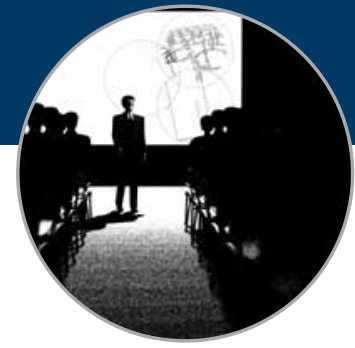

available, but a key point is that bioanalysts will have to decide the most appropriate way to utilize it. Korfmacher demonstrated how easy it is to remove unwanted background signals from HRMS spectra to find the peak of interest, using sidenafil as an example.

There are currently two types of HRMS systems available (FIGURE 2A \& B) - the LTQ Orbitrap ${ }^{\circledR}$ and the Waters quadrupole-Tof (QTof ${ }^{\mathrm{TM}}$ ). Both are excellent systems for metabolite identification; however, Kormacher believes that the Waters QTof system "will be most used for quantitative analysis in the future."

For quantitative analysis, the QTof system must have a scan rate of greater than $10 \mathrm{~Hz}$ (high scan rate is particularly important with UPLC ${ }^{\circledR}$ to ensure that there are sufficient points across the peak), mass resolution of greater than 30,000, mass accuracy greater than $5 \mathrm{ppm}$ and a linear dynamic range spanning at least three orders of magnitude. The two systems currently marketed by Waters meet these levels (Figure 2C \& D).

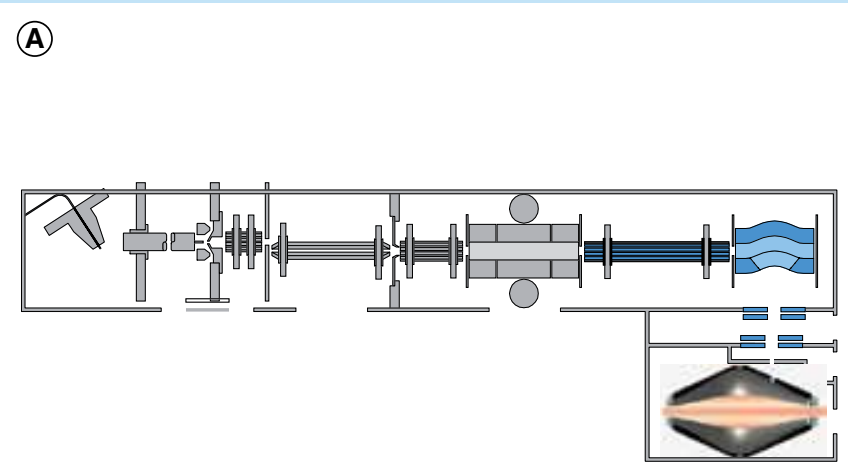

The LTQ Orbitrap ${ }^{\circledR}$ MS provides mass resolution of 10,000-100,000
(B)

\section{Quadrupole-time-of-flight}

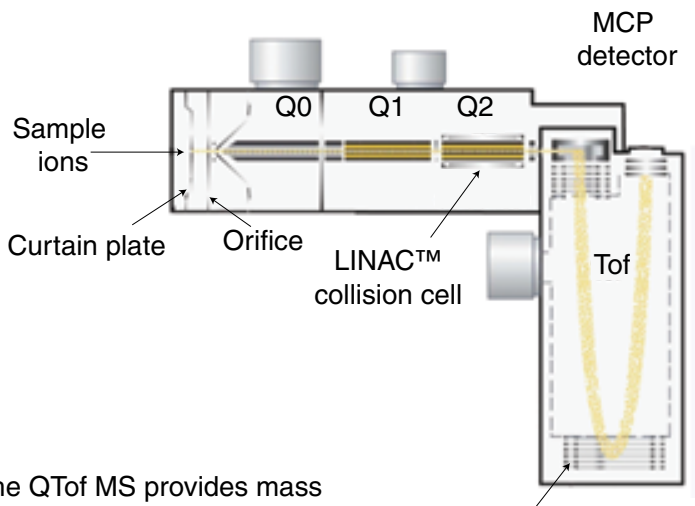

resolution of $10,000-50,000$
Ion mirror

(C)
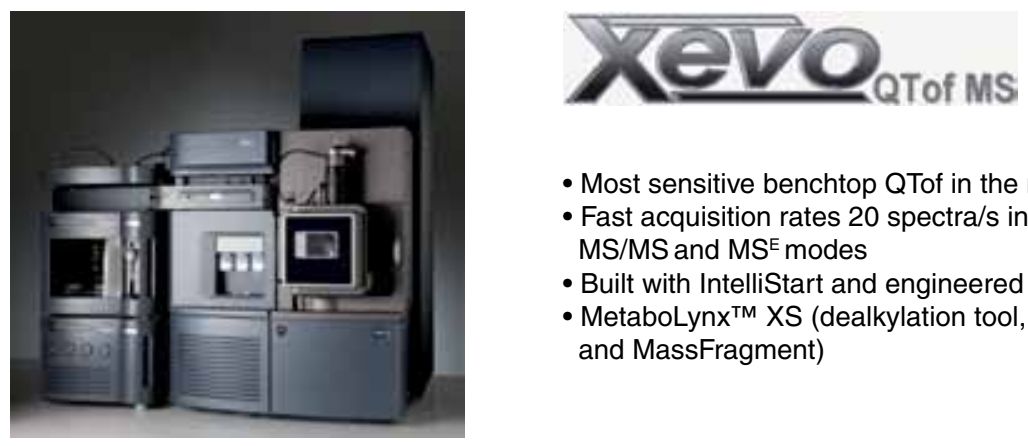

- Most sensitive benchtop QTof in the market

- Fast acquisition rates 20 spectra/s in MS,

MS/MS and MS ${ }^{\mathrm{E}}$ modes

- Built with IntelliStart and engineered simplicity

- MetaboLynx ${ }^{T M}$ XS (dealkylation tool, inteligent MDF and MassFragment)

(D)
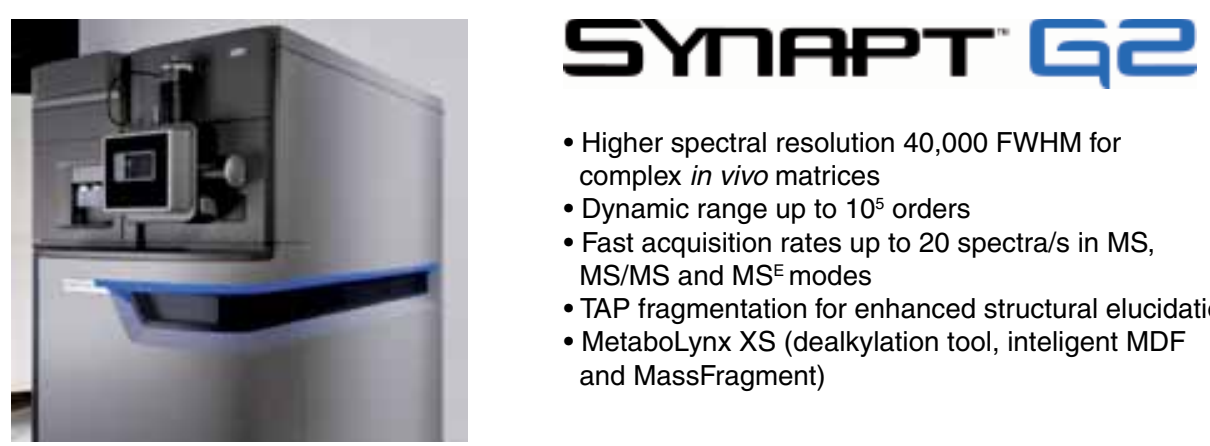

- Higher spectral resolution 40,000 FWHM for

complex in vivo matrices

- Dynamic range up to $10^{5}$ orders

- Fast acquisition rates up to 20 spectra/s in MS, MS/MS and MSE modes

- TAP fragmentation for enhanced structural elucidation

- MetaboLynx XS (dealkylation tool, inteligent MDF and MassFragment)

Figure 2. High-resolution MS systems. (A) LTQ Orbitrap ${ }^{\circledR}$, (B) QTof, (C) Waters Xevo ${ }^{\circledR}$ QTof and (D) Waters SYNAPT ${ }^{\circledR}$ G2 MS. FWHM: Full width at half maximum; MCP: Microchannel plate; MDF: Mass-defect filter; QTof: Quadrupole-time-of-flight; TAP: Time-aligned parallel. 
The workflow on these systems is very simple: the samples are injected onto UPLC linked to a HRMS and collect full scan data with no method development required. Once the data has been collected, the software utilized is very important; the systems will not work optimally with the wrong software. This is really what differentiates one system from another and should be a major consideration when purchasing your HRMS system. Once you have obtained the exact mass with your QTof, software such as Waters MetaboLynx ${ }^{\mathrm{TM}} \mathrm{XS}$ can be used to interrogate the data. Software such as this includes:

- Mas-defect filtering, which finds metabolites based on the exact mass of the analyte;

- UPLC-MS ${ }^{\mathrm{E}}$, which automatically provides information on precursor and product ions;

- MassFragment ${ }^{\mathrm{TM}}$, which can assign product ions.

Korfmacher illustrated this with data from Waters on the model compound atorvastatin (Figure 3) where, using MetaboLynx XS, the HRMS data obtained for atorvastatin was easily interrogated to find four metabolites. Work carried out by Kate Yu et al. (Waters Corporation) was cited as another example where the drug was incubated in solution and the metabolites generated were identified. So, the Xevo G2 QTof offers superb sensitivity and exact-mass measurement with high data-acquisition speed. The UPLC-Xevo G2 QTof $\mathrm{MS}^{\mathrm{E}}-$ MetaboLynx XS workflow allows rich information (both qualitative and quantitative) to be obtained from a single sample set with one injection per sample and metabolite internal diameter is performed in an easy workflow so that the working efficiency and productivity is greatly enhanced.

As another example, Korfmacher mentioned the Biocius RapidFire ${ }^{\circledR}$. RapidFire is a fast, integrated autosampler and SPE system that replaces the LC in LC-MS/MS. Eliminating the LC reduces the cycle time from $1-10 \mathrm{~min} / \mathrm{sample}$ to just 5-10 s/sample, is compatible with a range of biological matrices and works surprisingly well. However, the method-development time remained at 4-10 $\mathrm{min} /$ analyte using triple quadrupole MS. Switching to HRMS, they have eliminated the method-development bottleneck to enable rapid metabolite stability assays. For a final example of HRMS compared with triple quadrupole MS, Korfmacher cited a presentation given at this year's American Society for MS meeting involving a parallel artificial membrane permeability assay [2]. A correlation slope of almost one was achieved, demonstrating that HRMS works well for these type of protein-binding assays.

\section{"...the software utilized is very important; the systems will not work optimally with the wrong software."}

Mass spectrometry continues to be the most important tool used in discovery bioanalysis. In the past, different tools have been applied for metabolite identification and quantitative bioanalysis but Korfmacher believes that we are approaching a paradigm shift and that, in the future, HRMS will be used for both.

\section{- Questions to Walter Korfmacher}

If you need to use negative ionization, will it work?

The answer is yes. Most systems would be run in positive ion mode, which works fine in most cases. If it does not work, you can run it in negative ion mode and look for the laggards that did not show up in positive mode.

Your talk included a lot of references to software but I could probably access more software with my online forum than I can in the lab. Do you see this as one of the bottlenecks that we need to get through?

Software is the key. The hardware is easy in that the vendors are all going to make hardware tools that work well, but what we have found in our initial testing of some of the hardware is that the software just was not ready yet. The vendors are working on it, but I urge everyone that takes out an instrument demo to make sure you check out the software. Do not just let the vendors show you the data and take it from them that is easy to use because software is what makes it easy or hard. Without good software, the hardware will not help you. 


\section{News \& Analysis I Waters Bioanalysis World Tour}

(1)

III

(A) Atorvastatin results - MetaboLynx ${ }^{\mathrm{TM}} \mathrm{XS}$ browser

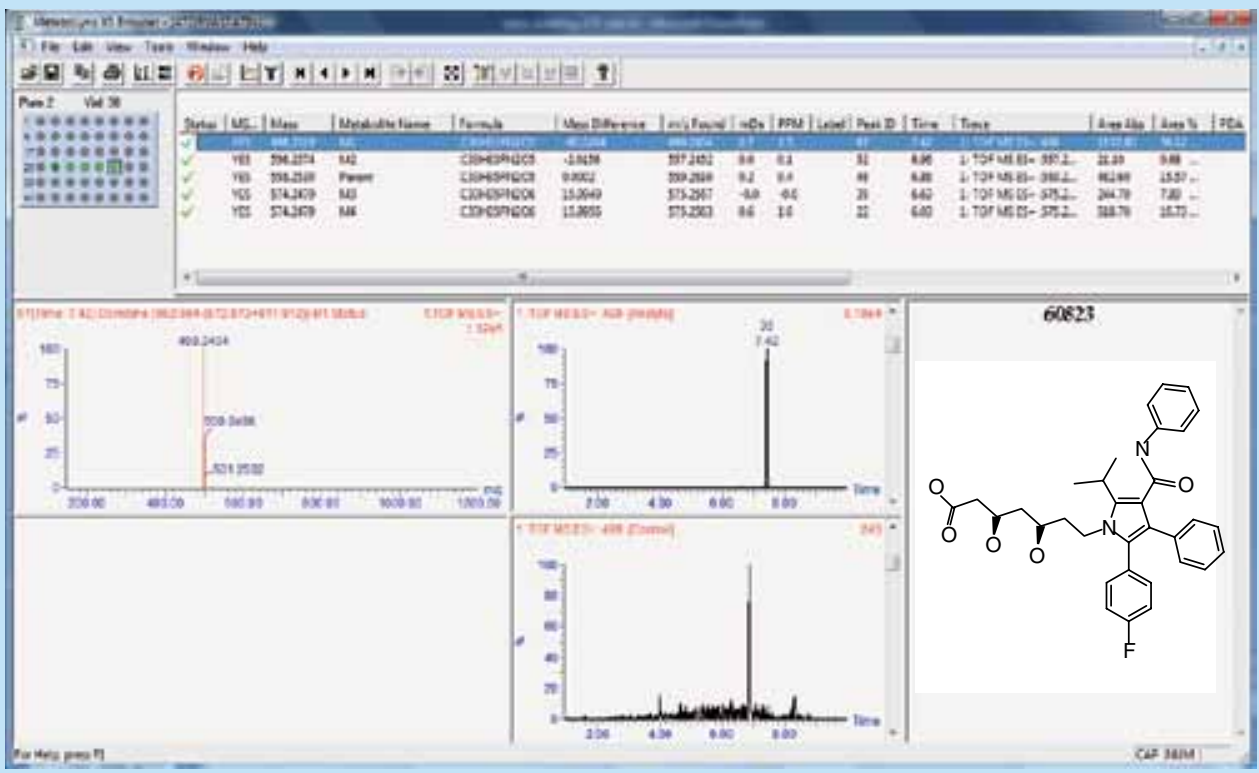

(B) Assignment of fragment ion of M1

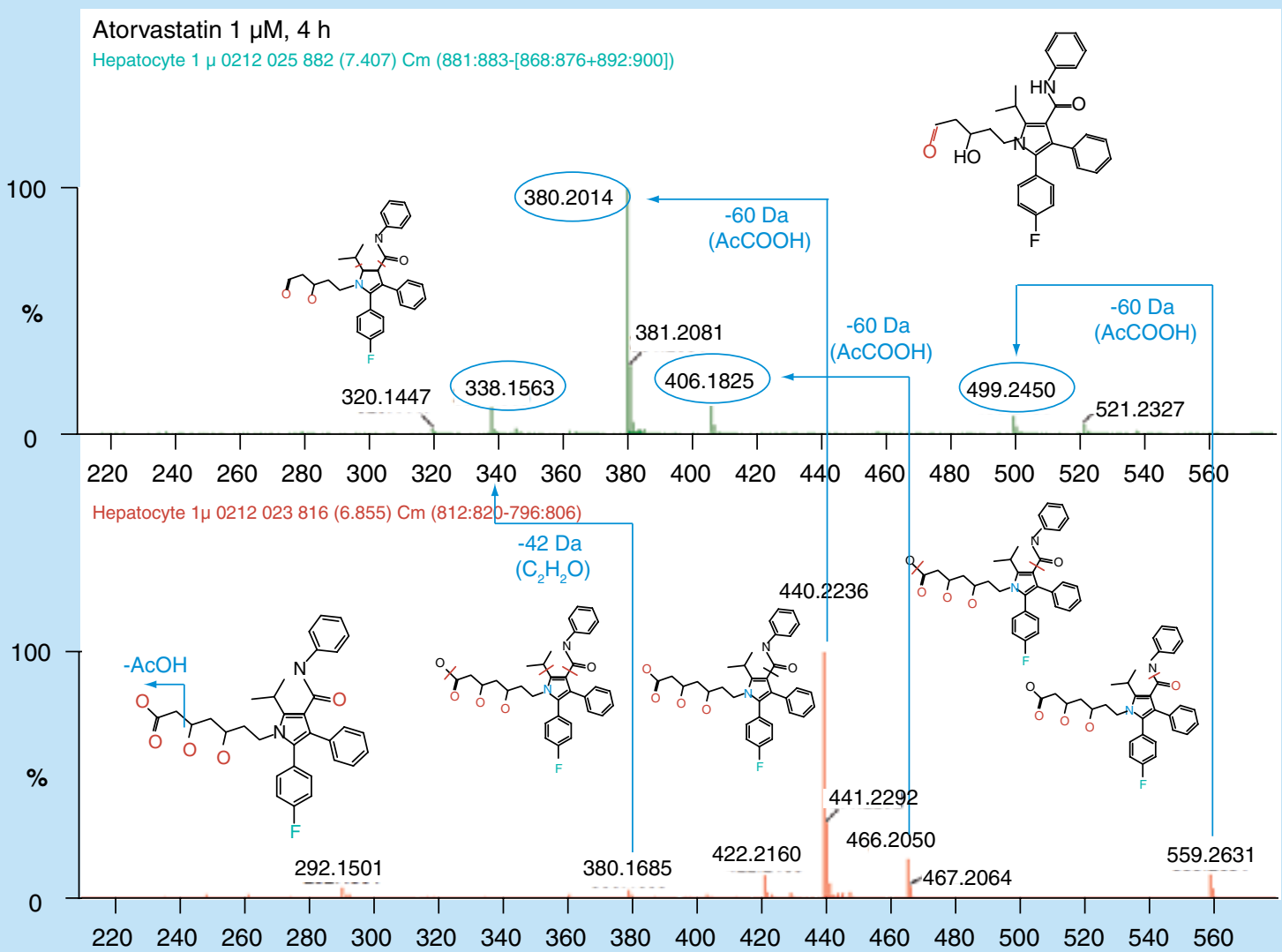

Figure 3. Interrogation of atorvastatin results. (A) MetaboLynx ${ }^{\mathrm{TM}} \mathrm{XS}$ screen shot. (B) Assignment of fragment ions. 
(C) Postulated metabolic pathways of atorvastatin

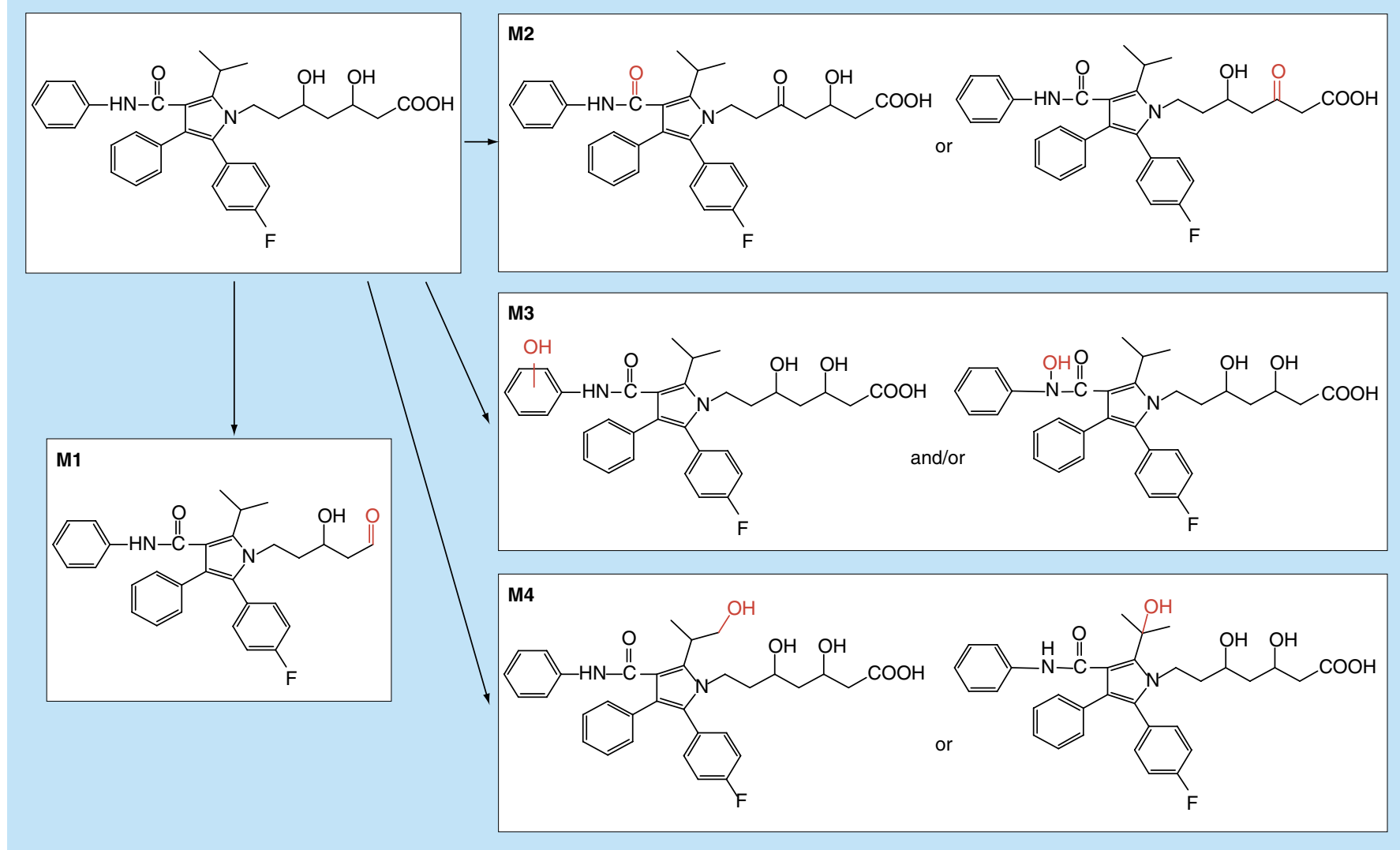

Figure 3 cont. Interrogation of atorvastatin results. (C) Postulated metabolic pathway.

\section{Integrated solutions for the future of bioanalysis}

Gary Schultz, Advion BioServices Inc., NY, USA

Gary Schultz's talk began by looking back to 1998 , when Advion purchased an accurate-mass LCT ${ }^{\mathrm{TM}}$ Tof mass spectrometer from Waters [3] to assess its performance against triple quadrupole mass spectrometers for drug-discovery quantitation, to demonstrate how far the technology has progressed in the last 10 years, before describing how he sees HRMS being applied in future bioanalysis work. Approximately 10 years ago, they decided that triple quadrupoles were superior to the accurate-mass instruments available at that time for performing multi-analyte bioanalysis, due to their sensitivity and linear dynamic range. Triple quadrupoles were tenfold higher in sensitivity than Tof-MS's limited dynamic range of $10^{2}-10^{3}$. Now in 2010, we have seen a dramatic improvement in the performance of accurate-mass instruments.
All the major instrument vendors now have HRMS available and competition between them is driving innovation forwards. However, there are differences between instruments. Schultz's presentation included data obtained using a Thermo Exactive Orbitrap ${ }^{\mathrm{TM}}$ and an AB Sciex TripleTof 5600 (Fıgure 4). With the Orbitrap, as the resolution increases so does the scan time ten scans/s gives a resolution of 10,000 but for a resolution of 50,000, two scans/s are acquired. With Tof instruments, the resolution is independent of scan speed. Overall, a resolution of $30,000-40,000$ is sufficient to quantify with good selectivity compared with today's triple quadrupoles. The amount of data generated with HRMS is so great that, as Korfmacher pointed out, good software tools are vital. 


\section{SYMPOSIUM REPORT I NEWS \& ANALYSIS}

identification with customizable, searchable libraries), Quick Calc (for discovery reporting for high-throughput screening assays) and Trace Analysis, which is a new application for determining known and unknown compounds. Looking at reserpine as an example (Figure 5), Schultz showed how the software can automatically assign structural information to the MS peaks.

"...the right software is key to getting the best out of exact-mass MS instrumentation."

A peptide biomarker method was validated. In a triple quadrupole system, this is challenging when the peptide multiply charges, one charge state is selected for MS/MS. Peptides tend to fragment to produce many product ions of equal intensity. With a triple quadrupole you have to pick one charge state and one product ion, and a lot of the data are discarded. With an accuratemass instrument, data on all charge states and isotopes is collected and can be summed and used for quantification.
Another important consideration is high resolution. An example was shown that demonstrated that, whilst both the triple quadrupole and the HRMS performed well, the accuratemass system had slightly better precision all the way down to the LOQ (TABLe I). The systems are already matched in precision, so the vendors have plenty of opportunity to improve.

Despite their advantages, HRMS systems come with their own challenges, not least the massive amount of data $-90 \mathrm{MB} /$ run compared with $540 \mathrm{~kb} / \mathrm{run}$ in a triple quadrupole. This makes data processing slower - tens of seconds rather than seconds to reintegrate a run. You also have to find somewhere to store the data and decide how to store it: currently, mass spectra versus time is kept, but for HRMS exact-mass chromatograms versus time can be stored giving you a complex 3D dataset.

\section{"The move to UPLC with smaller columns and more sensitive exact mass means that sample volumes can be greatly reduced."}

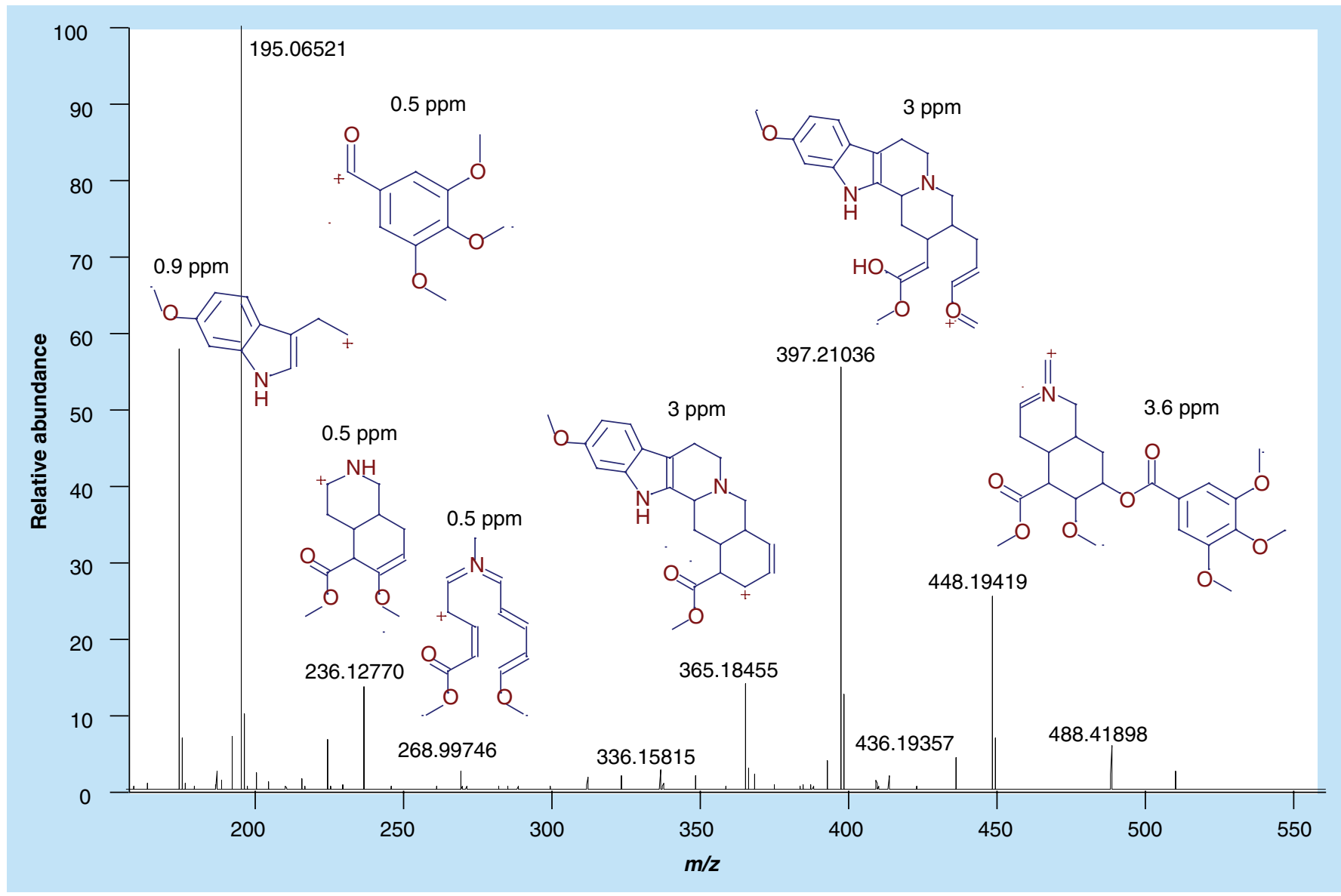

Figure 5. Exactive higher-energy collisional dissociation cell fragmentation of reserpine. 
accurate PK studies to be performed on a single rodent, rather than using pooled samples from multiple animals. Thus, we have seen a focus on DBS, which typically take $10-25 \mu$ of sample. Smaller sample volumes bring another set of analytical challenges, particularly with sensitivity and incurred sample reanalysis - do we have enough to re-assay? This is where microfluidic devices are important - coupled with QTof, you can obtain both quantitative and qualitative data in a single run. Sample handling is always going to be challenging with microlitre-sized samples as the more you handle them, the more chance you have of losing things or introducing contamination; this is particularly troublesome if you are looking for unknowns, such as with metabonomics studies.

\section{"Combining the microfluidic columns with}

QTof enables simultaneous quantitative and qualitative data acquisition, gives linear ranges of three to five and enables full scan precursor and fragment ion data to be collected in the same run..."

Rainville's work over the past year has involved microfluidic bioanalysis in the search for metabonomic biomarkers. Rainville and his colleages at Imperial College (London, UK) have been applying microfluidic columns to the investigation of biomarkers in axenic rats dosed with hydrazine [5]. Axenic rats are born by Caesarean section and are then kept in a sterile environment, so they do not have any gut microflora within them. In metabonomics experiments, many of the metabolites that you detect are due to metabolism by the gut microflora and not what is actually happening within, for example, the liver. So, the effect of the gut microflora on the metabolic profile before and after exposure to the model toxin hydrazine can be determined. Conventional and axenic rats dosed with vehicle alone, 1 or $60 \mathrm{mg} / \mathrm{kg}$ of hydrazine were studied.

The microfluidic column used was a prototype ceramic device packed with $1.7-\mu \mathrm{m}$ particles coupled to a QTof, for similar reasons highlighted by Korfmacher and Schultz. The 300$\mu \mathrm{m}$ geometry microfluidic device is shaped like a question mark (FIGURE 6), as it is compact and easy for customers to use. Furthermore, almost any geometry is possible by cutting the desired internal diameter by laser. Fittings can be a challenge with capillary nanoscale chromatography - inexperienced analysts can have trouble cutting the fittings correctly, resulting in voids that can deteriorate the chromatographic performance.
The planar plug-and-play devices don't have these connection problems. With sub-2- $\mu \mathrm{m}$ particles, the microfluidic devices exhibit higher peak capacities and narrower peaks, resulting in less co-elution and matrix effects. As the injection volumes proportional to the column are still relatively large, your cycle times can be sped up by increasing the flow rate. A lot of work has been done with the MS source making sure that there is no interference from the outside and to produce a stable spray at reduced capillary voltages from 0.8 to $2.4 \mathrm{kv}$, which may be more optimum for certain compounds undergoing quantification.

\section{"...QTof technology is able to carry out} quantitative and qualitative analysis in a single run, which maximizes the data obtainable from small samples."

Combining the microfluidic columns with QTof enables simultaneous quantitative and qualitative data acquisition, gives linear ranges

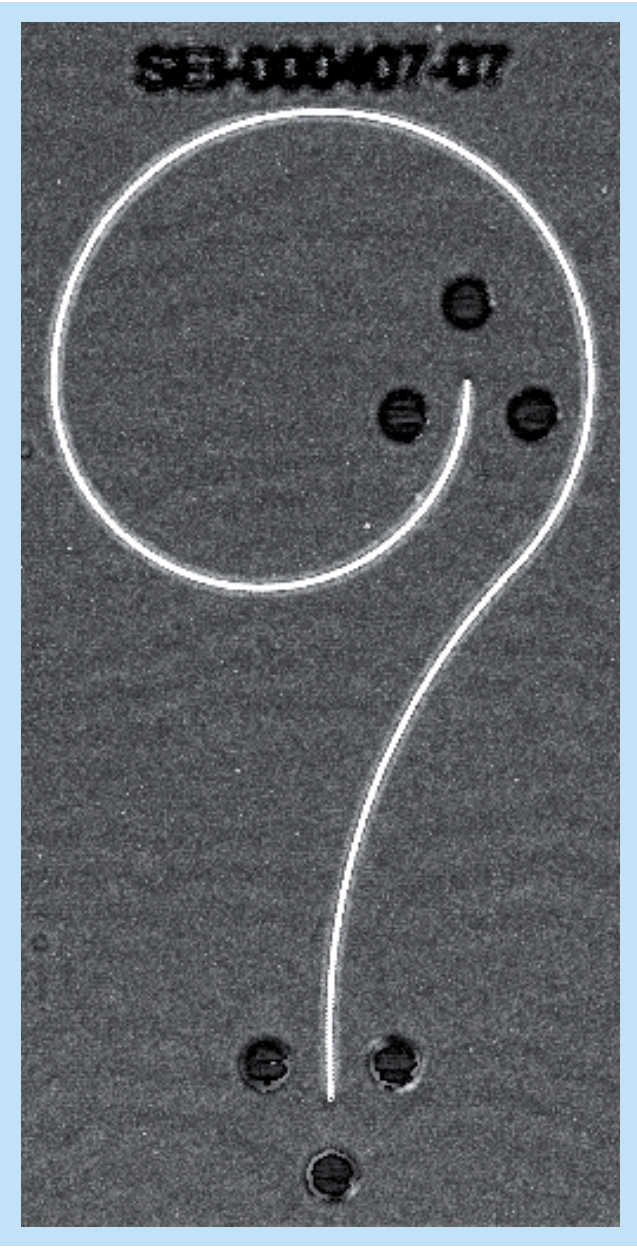

Figure 6. Ceramic microfluidic device. 


\section{News \& Analysis I Waters Bioanalysis World Tour}

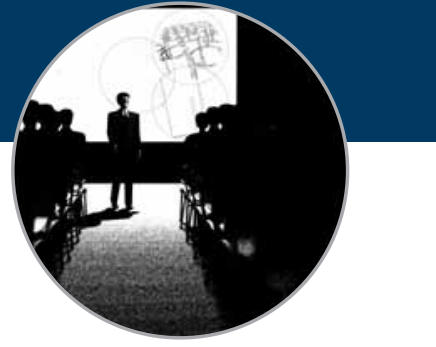

(A)

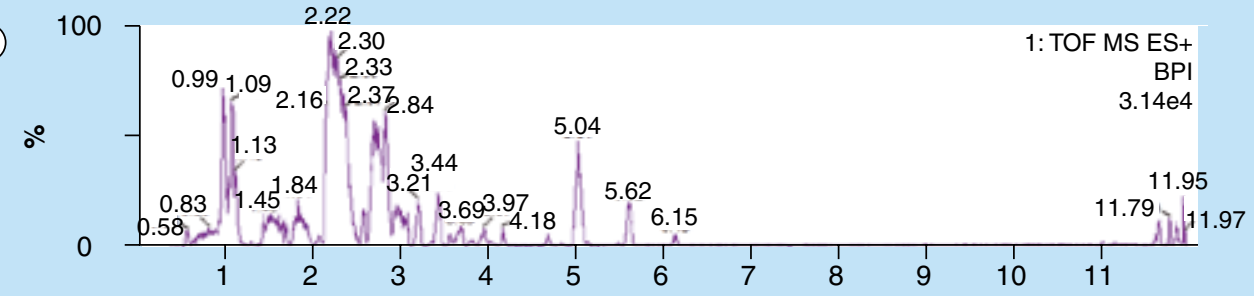

(B)

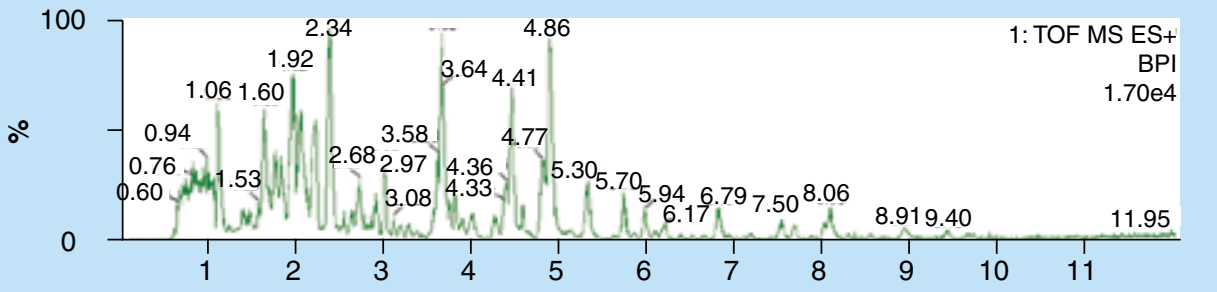

(C)
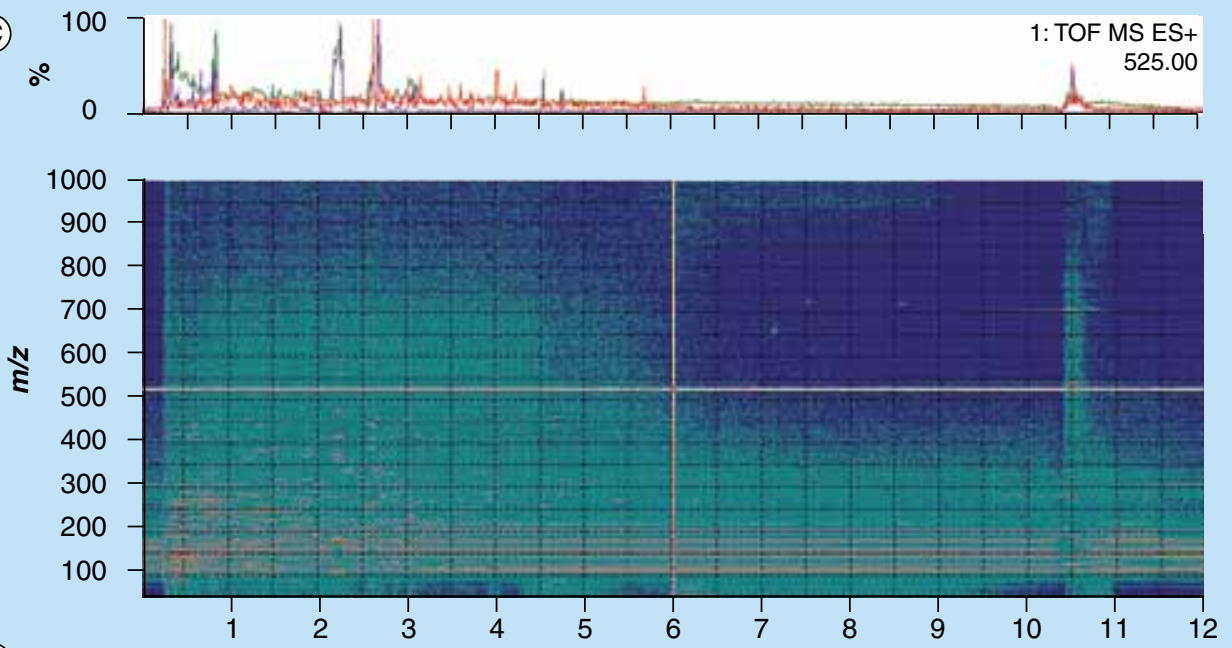

(D)
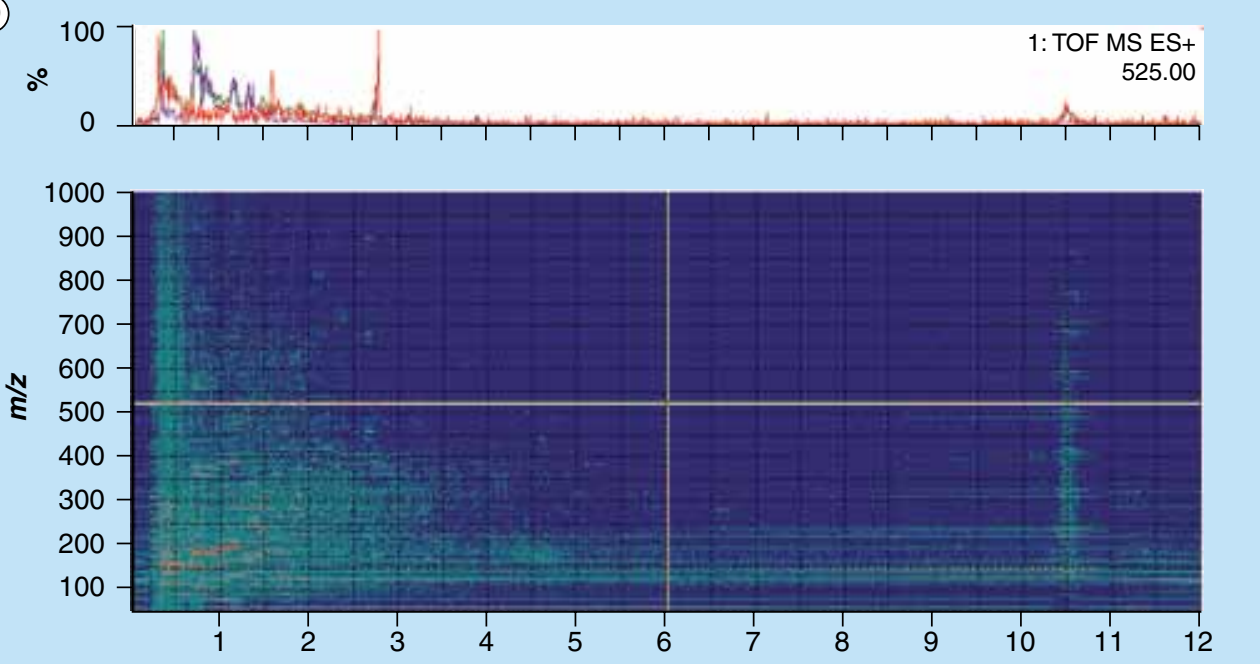

Figure 7. Analysis from hydrazine-dosed axenic rat with different $\mathrm{pH}$ mobile phases. Chroamtograms from (A) acidic and (B) basic mobile phases. MAP plots from (C) acidic and (D) basic mobile phases.

of three to five and enables full scan precursor and fragment ion data to be collected in the same run. Waters $\mathrm{MS}^{\mathrm{E}}$ technology means that collision cells can alternate between high and low collision energy to get fragmentation and parent ion information. Exact-mass MS enables the analyte detection window to be narrowed, thus reducing interfering compounds that might otherwise be clumped in with the metabolite or biomarker of interest. 
Rainville compared a 1- $\mu$ l sample injection carried out on a UPLC and a microfluidic device. Theoretically, you would expect a 50-fold increase in sensitivity when reducing the column diameter from $2.1 \mathrm{~mm}$ to $300 \mu \mathrm{m}$; in reality, there was a 20 - to 40 -fold increase, which is still very impressive. Using QTof data and the knowledge from previous studies of the identity of some of the biomarkers from hydrazine-dosed rats, the $M S^{\mathrm{E}}$ data can be interrogated. The low collision energy data gives the exact mass and the high collision-energy data confirms the fragmentation pattern.

The $\mathrm{pH}$ stability of the microfluidic columns is very important to allow both positive and negative electrospray ionization to be done. Approximately $85 \%$ of Rainville's work is positive electrospray, but different $\mathrm{pHs}$ can be useful in both positive and negative electrospray modes. Figure 7A shows the separation of a hyrazine-treated mouse sample run under acidic mobile-phase conditions, while Figure 7B shows the same sample run with basic mobile-phase conditions. What one observes is that although the acidic conditions produced a higher overall signal in the mass spectrometer under ESI positive conditions, the result produced under the basic conditions yields a higher resolution chromatogram. Figure 7C shows a MAP plot of the run under acidic conditions and Figure 7D the MAP plot under basic conditions. Observed here are the constant visible ions present (in orange) under acidic conditions. The constant presence of these ions could cause ion suppression that may lead to the inability to detect low abundant biomarkers present in the sample. The point here is that although acidic mobile phases are traditional with positive ESI MS analysis, the use of basic mobile phases may have utility when one is analyzing complex samples and is trying to define all constituents in the sample. An additional advantage of the utilization of high $\mathrm{pH}$ mobile phases is the reduction in chromatographic peak tailing for basic compounds.

Once you have obtained a metabonomics dataset, you need to interpret it using partial least squares, principle component analysis or software such as Waters MarkerLynx ${ }^{\mathrm{TM}}$ to make groupings and rationalize the data. In the case of hydrazinedosed rats, many metabolites were found that were not associated with the gut microflora, so these are definitely associated with the hydrazine. The next step is to match the marker to the biology - for example, one of the markers found, 2-aminoadipate, is not detected in control rats and we can see that this marker actually blocks one of the biochemical pathways following hydrazine dosing.

In conclusion, Rainville showed that QTof technology is able to carry out quantitative and qualitative analysis in a single run, which maximizes the data obtainable from small samples. The ceramic microfluidic devices resulted in a very sensitive analytical method and provided a robust method capable of running samples in both acidic and basic conditions without any deleterious effects from the chromatography. Finally, basic mobile-phase run in positive ionization mode can lead to additional biomarker identification.

\section{Conclusion}

To conclude the symposium, Chair Lowes showed his appreciation to the three speakers and highlighted that, having discussed high-resolution exact-mass applications and software evolution, in 10 years time today's software will be unrecognizable. Reduced sample sizes, microfluidic devices and nanospray applications are all going to be really important in the future. There were a lot of synergies between the three speakers and a lot of great ideas regarding where they think we are going to be in 10 years time. At the end of 2020, it will be interesting to look back at what we envision today and how it all plays out on a global stage within our rapidly changing pharmaceutical industry.

The Waters Bioanalysis World Tour continues throughout 2011, with the next event to be held at the Applied Pharmaceutical Analysis (Hyderabad, India). Full details can be found on the World Tour website [101].

\section{Free article downloads}

We are delighted to offer readers of this article three complimentary article downloads of papers relevant to the topics discussed in this symposium from Bioanalysis.

Please go to www.future-science-group.com/m/325 and enter the code AAPS2010 to receive free access to the following papers:

Yuan W, Edwards JL. Capillary separations in metabonomics. Bioanalysis 2(5), 935-963 (2010).

Plumb RS, Mather J, Little D et al. A novel LC-MS approach for the detection of metabolites in DMPK studies. Bioanalysis 2(10), 1767-1778 (2010).

Varma D, Jansen SA, Ganti S. Chromatography with higher pressure, smaller particles and higher temperature: a bioanalytical perspective. Bioanalysis 2(12), 2019-2034 (2010). 
